\title{
LRG ameliorates steatohepatitis by activating the AMPK/mTOR/SREBP1 signaling pathway in C57BL/6J mice fed a high-fat diet
}

\author{
TAO HAO ${ }^{1,2}$, HONGYING $\mathrm{CHEN}^{3}$, SISI WU $^{3}$ and HAOMING TIAN ${ }^{1}$ \\ ${ }^{1}$ Department of Endocrinology and Metabolism, West China Hospital, Sichuan University, Chengdu, Sichuan 610041; \\ ${ }^{2}$ Department of Endocrinology and Metabolism, The First People's Hospital of Dujiangyan, Dujiangyan, Sichuan 611830; \\ ${ }^{3}$ Core Facility, West China Hospital, Sichuan University, Chengdu, Sichuan 610041, P.R. China
}

Received September 6, 2018; Accepted April 25, 2019

DOI: $10.3892 / \mathrm{mmr} .2019 .10304$

\begin{abstract}
The pathogenesis of nonalcoholic fatty liver disease non-alcoholic steatohepatitis (NASH) has not been fully elucidated, and there are currently no effective treatments for NASH. The aim of the present study was to explore the therapeutic effects of the glucagon-like peptide-1 (GLP-1) receptor agonist liraglutide (LRG) on NASH and the underlying mechanisms. C57BL6J mice were fed a high-fat diet (HFD) for 8 weeks to induce hepatic steatosis, and then LRG was injected subcutaneously for 4 weeks. The expression of sterol regulatory element-binding protein 1 (SREBP1) and adenosine monophosphate-activated protein kinase (AMPK) as well as the phosphorylation of mechanistic target of rapamycin (mTOR) and p70 ribosomal S6 kinase (p70S6K) were determined by western blot analysis. The intracellular distribution of SREBP1 was assessed by immunofluorescence staining. The results revealed that LRG treatment ameliorated HFD-induced hepatic lipid accumulation and inhibited body weight gain. In addition, LRG treatment significantly suppressed the expression of hepatic SREBP1 as well as the phosphorylation of mTOR and p70S6K; it also increased the phosphorylation of AMPK and acetyl coenzyme A carboxylase. Furthermore, LRG treatment inhibited the hepatic nuclear translocation of
\end{abstract}

Correspondence to: Professor Haoming Tian, Department of Endocrinology and Metabolism, West China Hospital, Sichuan University, 37 Guoxue Road, Chengdu, Sichuan 610041, P.R. China E-mail: hmtian999@126.com

Abbreviations: ACC, acetyl-CoA carboxylase; AMPK, AMP-activated protein kinase; GLP-1, glucagon-like peptide-1; H\&E, hematoxylin and eosin; HFD, high-fat diet; mTOR, mechanistic target of rapamycin; NAFLD, nonalcoholic fatty liver disease; SREBP1, sterol regulatory element-binding transcription factor 1

Key words: glucagon-like peptide 1, liraglutide, nonalcoholic fatty liver disease, steatohepatitis, sterol regulatory element-binding protein 1 , mechanistic target of rapamycin
SREBP1. It was suggested that the GLP-1 receptor agonist LRG may have ameliorated hepatic steatosis by activating the AMPK/mTOR/SREBP1 signaling pathway as opposed to inhibiting body weight gain.

\section{Introduction}

Nonalcoholic fatty liver disease (NAFLD) is the most widespread liver disease worldwide, and its incidence continues to rise $(1,2)$. It describes a number of livers diseases including simple steatosis or NAFL with low inflammation, and can progresses to non-alcoholic steatohepatitis (NASH). NASH is the most severe form of NAFLD and is characterized by the presence of an abnormal accumulation of fat in the liver which can progress to liver cell injury (hepatocellular ballooning) and inflammation (3). NASH results from aberrant hepatic lipid accumulation, which is strongly associated with high-fat diet (HFD)-induced metabolic abnormality. A continuous intake of HFD contributes to the progression of NAFLD $(4,5)$. However, the exact molecular mechanisms underlying NASH remain largely unknown, and currently there are no effective therapeutic strategies for NASH apart from caloric restriction (CR) and regular exercise $(6,7)$. Therefore, further understanding of the pathology of NASH is critical in order to develop effective management strategies for NAFLD.

Sterol regulatory element-binding protein-1 (SREBP1) mediates the expression of lipogenesis-associated triglyceride synthesis and accumulation $(8,9)$. SREBP1 can cause excessive triglyceride accumulation in the liver, thereby leading to NAFLD development (10). Mechanistic target of rapamycin (mTOR) is a member of the phosphatidylinositide3-kinase-associated family of kinases and forms two distinct complexes: mTORC1 and mTORC2 (11). mTORC1 signaling stimulates cell growth via multiple mechanisms, including promoting lipid biosynthesis $(12,13)$. In addition, mTORC1 enhances de novo lipogenesis by enhancing the nuclear localization and activity of SREBP1 (14-16). Therefore, agents targeting mTORC1 have therapeutic potential for NASH.

Glucagon-like peptide-1 (GLP-1), an incretin hormone, as well as glucose-dependent insulinotropic polypeptide, are responsible for mediating glucose-mediated insulin production 
in pancreatic $\beta$-cells (17-19). GLP-1 and its analogues also perform pleiotropic functions in extra-pancreatic organs in mammals, including hepatic lipid deposition alleviation, weight loss and appetite inhibition $(20,21)$. GLP-1 may regulate the expression of genes associated with lipid metabolism in liver cells, thereby preventing the development and progression of NAFLD (22). Therefore, the GLP-1 receptor agonist liraglutide, may have potential for improving NASH outcomes as a novel therapeutic agent by activating the adenosine monophosphate-activated protein kinase (AMPK)/mTOR/SREBP1 signaling pathway.

\section{Materials and methods}

Animals. A total of 32 male C57/BL6J mice (18-20 g), 8 weeks of age, were supplied by the Laboratory Animal Center, West China Hospital, Sichuan West China School of Medicine (Chengdu, China). The mice were housed individually in cages at $20-25^{\circ} \mathrm{C}$ with a constant humidity $(55 \pm 5 \%)$ with a 12-h light/dark cycle. All animal experiments were approved by the Ethics Committee of Sichuan University (Sichuan, China) and were performed in accordance with guidelines of the Institutional Animal Ethics Committee and international guidelines $(23,24)$.

Animal groups and treatments. C57BL/6J mice were randomly divided into 4 groups ( $\mathrm{n}=8$ /group) as follows: Standard-fat diet: i) Control ( $8 \mathrm{kcal} \%$ fat, $42 \mathrm{kcal} \%$ protein and $50 \mathrm{kcal} \%$ carbohydrate); the HFD-fat diet groups: ii) the ad libitum group [61 kcal \% fat, $15 \mathrm{kcal} \%$ protein, and $25 \mathrm{kcal} \%$ carbohydrate, $0.2 \%$ cholesterol, high glucose water (2\% fructose plus $2.5 \%$ glucose) ad libitum], iii) the ad libitum+LRG (liraglutide, $0.6 \mathrm{mg} / \mathrm{kg} / \mathrm{day}$ ) and iv) the CR group (calorically restricted to follow the food intake trajectory of the ad libitum+LRG group). Liraglutide (Novo Nordisk, Oslo, Norway) was intraperitoneally injected into the mice in the LRG group every day while saline was injected into the other groups every day for 4 weeks. In all experiments, the body weight and food intake of the mice were determined twice per week. Animals were sacrificed at the end of week 12 , and the liver tissues were excised, immediately frozen in liquid nitrogen and stored at $-80^{\circ} \mathrm{C}$ for protein extraction.

Biochemical indices. Tail blood of mice was collected at 0, 3, $6,9,12$ weeks and was measured using a blood glucose meter (Life Scan. Inc., USA). After sacrificing the mice at the end of week 12 , serum was collected by centrifugation at $4,000 \mathrm{x} \mathrm{g}$ for $15 \mathrm{~min}$ at $4^{\circ} \mathrm{C}$. Serum levels of glutamic-oxaloacetic transaminase 1 (GOT1)/aspartate aminotransferase (AST), glutamic-pyruvate (GPT)/alanine aminotransferase (ALT) and total cholesterol (T-CHO) were assessed using an automatic biochemistry analyzer (AU2700; Olympus Ltd., Japan). Serum triglyceride (TG) levels were measured with ELISA kits (cat. no. BC0625; Solarbio Ltd., Beijing, China) according to the manufacturer's instructions.

Hematoxylin and eosin $(H \& E)$ and Oil red $O$ staining. Following perfusion with phosphate-buffered saline (PBS), the livers were removed for staining. For H\&E staining, the livers were fixed with $4 \%$ paraformaldehyde, embedded in paraffin, cut into $8 \mu \mathrm{m}$ sections, and then stained with H\&E. For Oil red $\mathrm{O}$ staining, the samples were embedded in optimal cutting temperature medium (Leica Microsystems GmbH, Germany), frozen and then cut into sections with a microtome. The frozen sections were stained with Oil red O.

Western blot analysis. Protein expression and phosphorylation were assessed by western blotting. The following antibodies from Cell Signaling Technology, Inc. (Danvers, MA, USA) were used: AMPK (cat. no. \#2795; dilution at 1:1,000), phosphorylated(p)-AMPK $\alpha$ (Thr172) (cat. no. \#8208; dilution at 1:1,000), mTOR (cat. no. \#2983; dilution at 1:1,000), p-mTOR (Ser2448) (cat. no. \#5536; dilution at 1:1,000), S6 (cat. no. \#2217 dilution at 1:1,000), p-S6 (Ser240/244) (cat. no. \#5364; dilution at 1:1,000), P70S6K1 (cat. no. \#9234s; dilution at 1:1,000), p-P70S6K1 (Thr389) (cat. no. \#2708; dilution at 1:1,000), acetyl coenzyme A carboxylase (ACC; cat. no. \#3662; dilution at 1:1,000), and p-ACC (Ser79) (cat. no. \#11818; dilution at 1:1,000). The anti-SREBP1 (cat. no. \#ab28481; dilution at 1:500) antibody and anti-GAPDH (\#ab9584; dilution at 1:10,000) were from Abcam (Cambridge, MA, USA), and secondary HRP goat anti-rabbit IgG antibodies (\#511202; dilution at 1:5,000) was from Zen BioScience (Chengdu, China).

Immunofluorescence assays. The liver sections were incubated with anti-SREBP-1 antibody (cat. no. \#ab28481; Abcam; $5 \mu \mathrm{g} / \mathrm{ml}$ ) and $1 \%$ goat serum (cat. no. \#ab7481; Abcam) at $4^{\circ} \mathrm{C}$ and 1:1,000 dilution overnight in PBS. Following washing with PBS, the sections were incubated with the secondary antibody (1:1,000; as aforementioned) at room temperature for $1 \mathrm{~h}$. Next, the sections were stained with 4',6-diamidino-2phenylindole (DAPI) for $5 \mathrm{~min}$ at room temperature, fixed, washed extensively with PBS, and then imaged under a fluorescence microscope (Axio Imager A2; Carl Zeiss, Jena, Germany) at x200 magnification.

Statistical analysis. Statistical analysis was performed using GraphPad Prism 6 software (GraphPad Software, Inc., La Jolla, CA, USA). The results are expressed as the mean \pm standard error. A Student's t-test or paired Student's t-test were used to compare two groups. Comparisons among multiple groups were analyzed using one-way analysis of variance (ANOVA) followed by the Scheffe post-hoc test. $\mathrm{P}<0.05$ was considered to indicate a statistically significant difference.

\section{Results}

Effects of LRG treatment on body weight, energy intake and blood glucose in C57 mice. The body weight and energy changes as well as blood fasting glucose in the different groups are presented in Fig. 1. To confirm the beneficial metabolic effects of LRG treatment in vivo, the CR group was designed in the present experiments to consistently match the energy intake of the LRG-treated group, thereby avoiding the effects of long-term LRG treatment on energy intake.

The body weights of the C57 mice fed the HFD both treated with LRG $(0.6 \mathrm{mg} / \mathrm{kg} / \mathrm{day})$ and the CR group (ad libitum+LRG and CR groups) were significantly lower than that of the ad libitum group ( $\mathrm{P}<0.01$ at 9 and 12 weeks); 

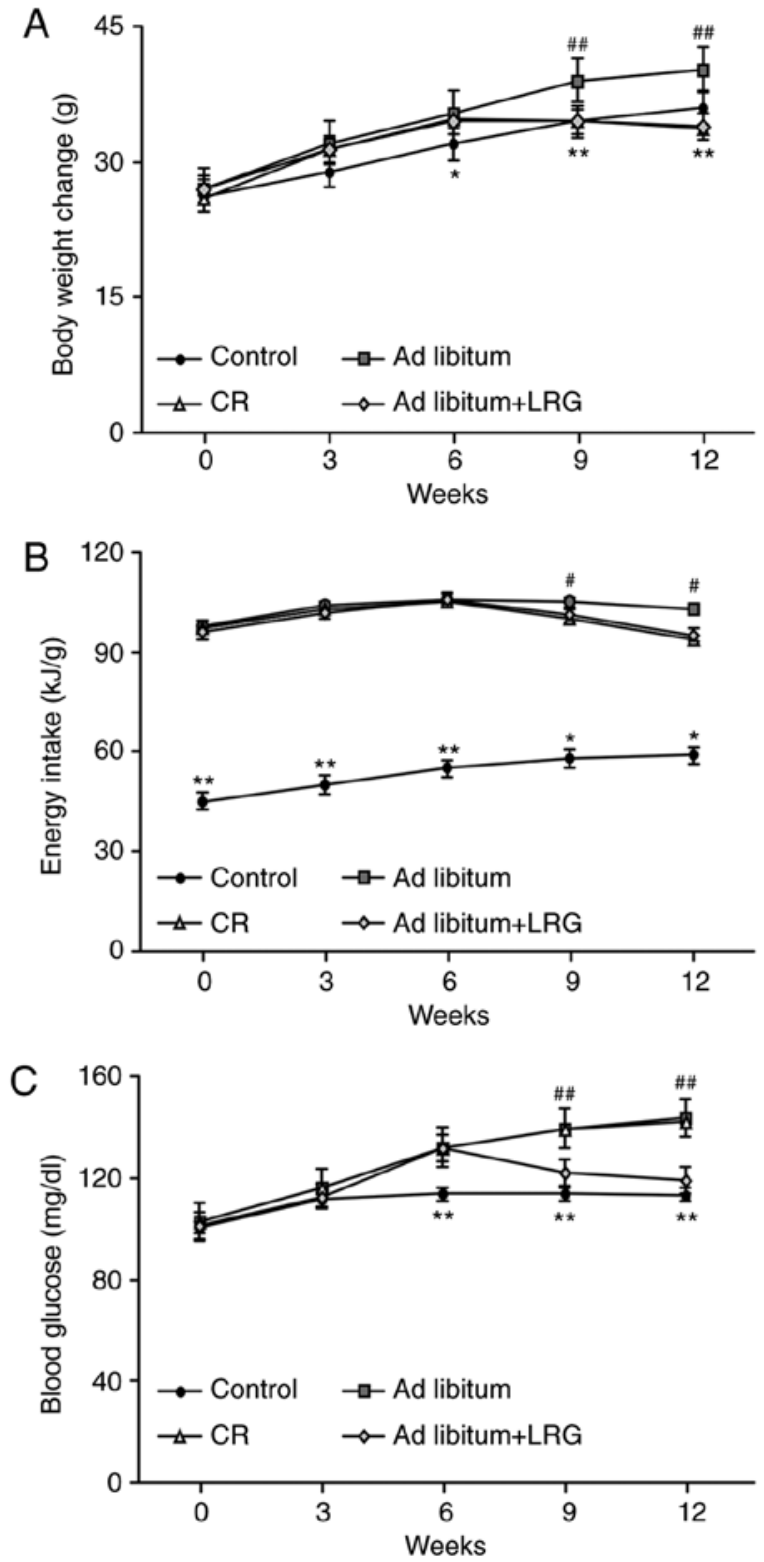

Figure 1. Changes in energy intake, body weight and fasting glucose of mice under different treatments. (A) Body weight changes, (B) energy intake and (C) fasting blood glucose levels in the different groups of mice during the 12-week experimental period. Values are expressed as the mean \pm standard error ( $\mathrm{n}=8$ animals/group). ${ }^{*} \mathrm{P}<0.05$ and ${ }^{* *} \mathrm{P}<0.01$ vs. the control group; ${ }^{\#} \mathrm{P}<0.05$ and ${ }^{\# \#} \mathrm{P}<0.01$ vs. the ad libitum group.

whereas there were no significant differences in whole body weights between the ad libitum $+\mathrm{LRG}$ and the $\mathrm{CR}$ group. The energy intakes of mice treated with LRG were significantly decreased when compared with the ad libitum $(\mathrm{P}<0.01$ at 9 and 12 weeks; Fig. 1B). No differences were observed in the energy intakes between the CR and ad libitum+LRG treatment groups. Furthermore, fasting blood glucose levels were significantly lower throughout the experimental period in the ad libitum+LRG group when compared with ad libitum and $\mathrm{CR}$ mice $(\mathrm{P}<0.01$ at 9 and 12 weeks; Fig. $1 \mathrm{C})$.

Effects of LRG treatment alleviates liver dysfunction and hepatic lipid accumulation in C57 mice. In comparison to the mice fed a standard diet (control), the liver weight, adjusted by body weight, was increased in the HFD-fed mice (ad libitum and CR groups; $\mathrm{P}<0.01$ ) compared to the control group; however, LRG treatment (ad libitum+LRG group) significantly decreased the liver weight when compared to the ad libitum group ( $\mathrm{P}<0.01$; Fig. $2 \mathrm{~A}$ and $\mathrm{B})$. In addition, liver function profiling demonstrated that the GOT1 and GPT were significantly decreased in the ad libitum+LRG group mice when compared with those in the ad libitum group $(\mathrm{P}<0.01$; Fig. $2 \mathrm{C}$ and $\mathrm{D})$; similar results were found for the levels of total cholesterol and triglycerides (Fig. 2E and F). Next, the present study performed H\&E and Oil red O staining (magnification, $\mathrm{x} 40$ ) of the liver sections from each group to visualize hepatic vacuole steatosis and lipid accumulation. Extensive micro-vesicular steatosis surrounding the perisinusoidal areas and lipid accumulation were observed in the HFD-fed mice; however, there was an apparent decrease in the amount of intracellular lipid droplets in the livers of mice in the ad libitum+LRG group (Fig. 2G and H).

Effects of LRG treatment on AMPK/mTOR/SREBP1 signaling in the liver of C57 mice. AMPK is a metabolic fuel gauge that regulates lipid metabolism by sensing changes in the intracellular AMP/adenosine triphosphate ratio, especially in the liver as reported by Liu et al (25). A canonical downstream mediator of AMPK is mTORC1. As shown in Fig. 3B, the level of phosphorylated (p)-mTOR in the ad libitum+LRG mice was significantly lower when compared with the ad libitum group $(\mathrm{P}<0.01)$, whereas was insignificant different compared with the CR group. As shown in Fig. 3A, LRG treatment significantly decreased the levels of p-S6 and p-p70S6K1, two conserved downstream targets of mTORC1, in the mice of the ad libitum and CR groups $(\mathrm{P}<0.01)$. When compared with the control, hepatic expression of p-AMPK was observed to be downregulated in the ad libitum and CR mice, while these levels were significantly restored in the ad libitum+LRG mice $(\mathrm{P}<0.01$; Fig. 3C). ACC, a downstream target of AMPK, is a key enzyme in fatty acid metabolism. Activated AMPK phosphorylates and inhibits ACC activity, leading to the inhibition of fat synthesis and increased oxidation (Fig. 4). The present study demonstrated that LRG treatment upregulated the phosphorylation of ACC and AMPK in the mice treated with HFD (Fig. 3A and C). SREBP1c promotes the synthesis of fatty acids and triglycerides (Fig. 4). As shown in Fig. 3B, the HFD-fed mice (ad libitum and CR groups) had significantly increased SREBP1 activity $(\mathrm{P}<0.01)$ when compared with the control group, yet this increase was significantly attenuated in the ad libitum+LRG mice when compared with the ad libitum group $(\mathrm{P}<0.05)$.

Effects of LRG treatment on the nuclear translocation of SREBP1 in the livers of mice. The present study further determined SREBP-1c expression and intracellular localization by conducting immunofluorescence staining of the liver tissues from each group (magnification, x200). There were significantly fewer hepatocytes positively stained with SREBP-1c in the ad libitum+LRG group compared with that observed in the mice in the ad libitum and $\mathrm{CR}$ mice $(\mathrm{P}<0.05)$. In addition, HFD led to the nuclear accumulation of SREBP1, which was inhibited by LRG treatment (Fig. 5). 

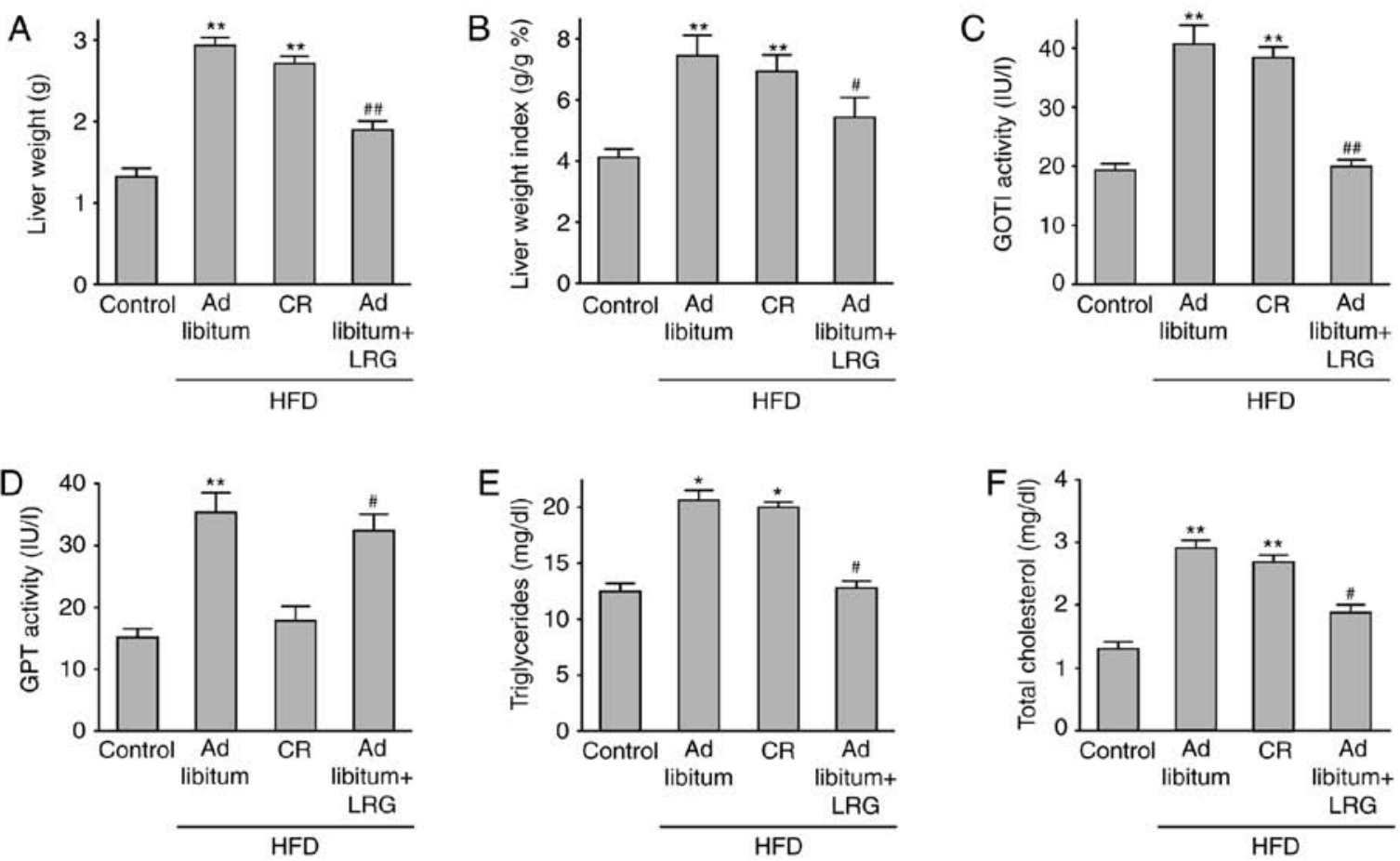

G
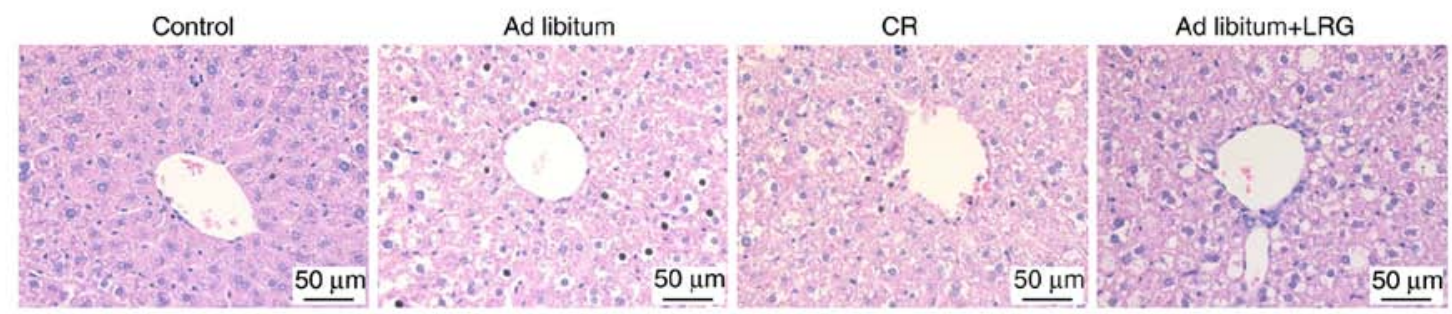

$\mathrm{H}$
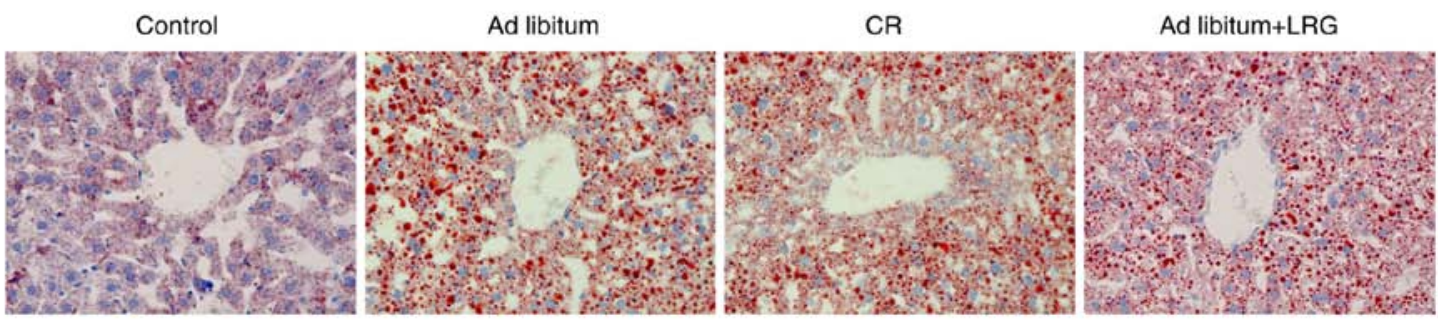

Figure 2. Effects of LRG treatment on hepatic function and hepatic lipid accumulation in HFD-fed mice. (A and B) LRG treatment significantly decreased the liver weight and liver-to-body weight ratio, $(\mathrm{C}$ and D) the GOT1 and GPT, and (E and F) the triglyceride and total cholesterol levels, which were increased in the HFD-fed mice. ( $\mathrm{G}$ and H) H\&E (magnification, $\mathrm{x} 40$ ) and oil red $\mathrm{O}$ (magnification, $\mathrm{x} 40$ ) staining in hepatic sections from the different groups revealed that hepatic vacuole steatosis and lipid accumulation were significantly increased in the HFD-fed mice and were decreased in the LRG-treated mice, indicating that LRG treatment alleviates the hepatic damage induced by the HFD. Values are expressed as the mean \pm standard error $\left(\mathrm{n}=8\right.$ animals/group). ${ }^{*} \mathrm{P}<0.05$ and ${ }^{* *} \mathrm{P}<0.01$ vs. the control group; ${ }^{\#} \mathrm{P}<0.05$ and ${ }^{\# \#} \mathrm{P}<0.01$ vs. the ad libitum group. LRG, liraglutide; GOT1, glutamic-oxaloacetic transaminase 1 ; GPT, glutamic-pyruvate transaminase; HFD, high-fat diet; H\&E, hematoxylin and eosin.

\section{Discussion}

The pathology of steatohepatitis (NASH) is largely unknown, and there are currently no effective treatments available for NASH N apart from diet and physical activity. In the present study, we investigated the therapeutic effects of the GLP-1 receptor agonist liraglutide (LRG) on NASH and the underlying mechanisms. The results revealed that C57 mice with HFD-induced NASH exhibited increased body weight and increased levels of hepatic fat. In addition, LRG treatment significantly reduced the body weight, improved hepatic lipid accumulation, and suppressed the elevated levels of total cholesterol and low-density lipoprotein cholesterol in the serum of HFD-fed mice. Mechanistically, it was revealed that LRG improves NASH through the AMPK/mTOR/SREBP1 signaling pathway.

AMPK is a sensor of intracellular energy status and negatively regulates mTORC1 (26-28). Recent studies have reported that AMPK agonists (such as metformin and adiponectin) improve NASH by inhibiting lipid synthesis via mTORC1/SREBP-1c signaling (12,29-31). In addition, ACC is a downstream target of AMPK and a rate-limiting enzyme involved in the synthesis of fatty acids. Activated liver AMPK inhibits fatty acid synthesis by increasing the phosphorylation and inactivation of ACC in order to reduce the production of malonyl coenzyme A $(32,33)$. In addition, mTORC1 triggers hepatic de novo lipogenesis to promote lipid synthesis by activating SREBP-1c $(26,33,34)$. 

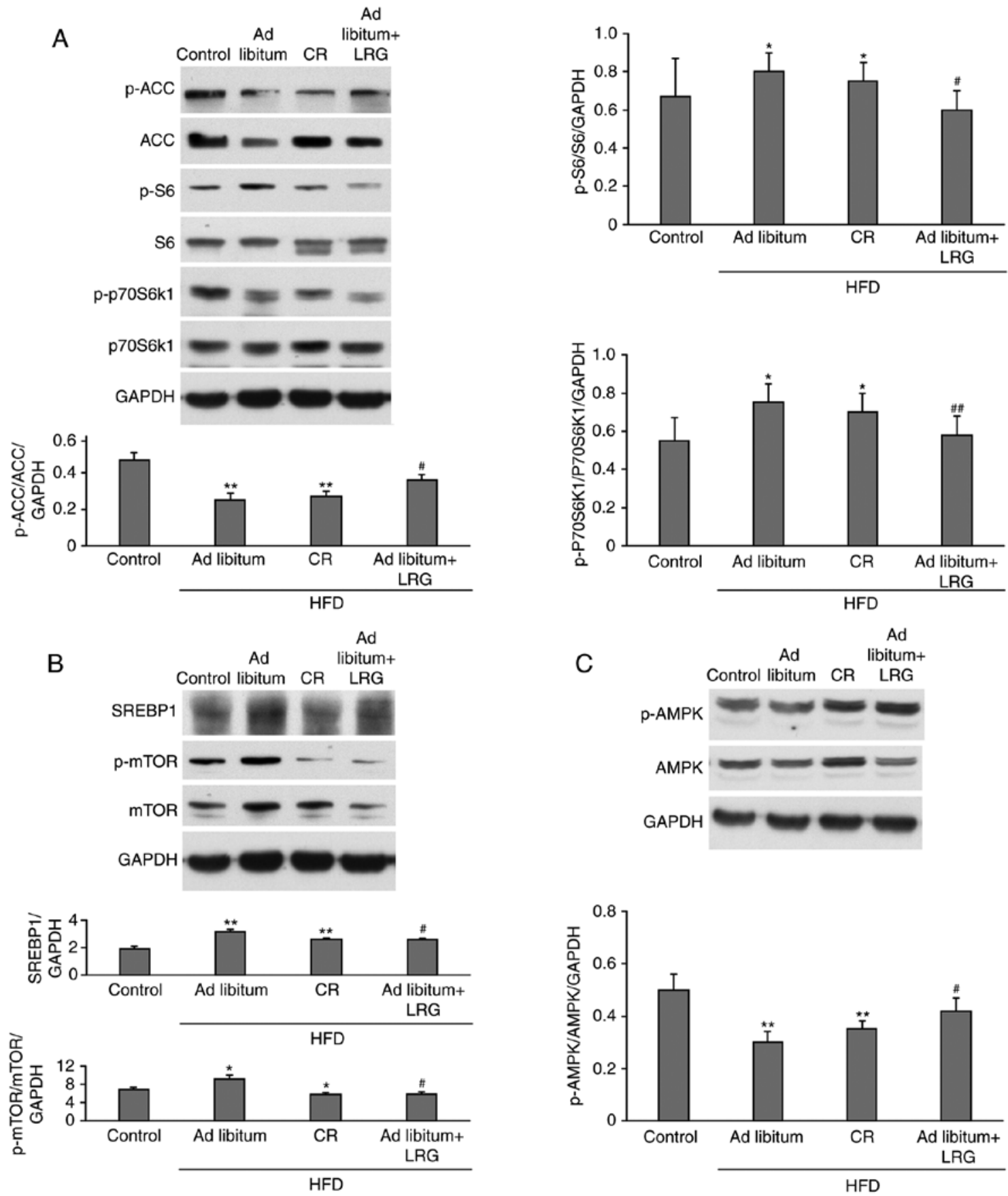

Figure 3. Effects of LRG treatment on the protein expression of AMPK/mTOR/SREBP1 in HFD-fed mice. C57 mice were fed a HFD for 12 weeks, and their liver tissues were then obtained. The protein expression levels of factors associated with lipogenesis [(A) ACC, p-ACC, S6, p-S6, p70S6k1, p-p70S6k1; (B) SREBP1, mTOR, p-mTOR; (C) AMPK, p-AMPK] were determined and compared between groups. Representative images of the western blotting assays of indicators associated with hepatic tissues are presented. Values are expressed as the mean \pm standard error ( $\mathrm{n}=8 /$ group). ${ }^{*} \mathrm{P}<0.05$ and ${ }^{* *} \mathrm{P}<0.01 \mathrm{vs}$. the control group; ${ }^{\#} \mathrm{P}<0.05$ and ${ }^{\# \#} \mathrm{P}<0.01$ vs. the ad libitum group. LRG, liraglutide; AMPK, adenosine monophosphate-activated protein kinase; mTOR, mechanistic target of rapamycin; SREBP1, sterol regulatory element-binding protein 1; HFD, high-fat diet.

Furthermore, mTORC1 regulates SREBP-1 activation by stimulating SREBP-1 mRNA expression, and promoting the nuclear localization and activity of SREBP-1 (30-32). Düvel et al (35) also demonstrated that $\mathrm{p} 70 \mathrm{~S} 6 \mathrm{~K} 1$ is required for the mTORC1-mediated increase and activation of SREBP1 (36-38). In the present study, LRG treatment reduced lipid accumulation in the liver by activating AMPK, thereby suppressing the mTORC1/SREBP1 signaling pathway.

The present results demonstrated that HFD inhibited the phosphorylation of AMPK in the mouse liver and significantly enhanced the phosphorylation of mTOR and $70 \mathrm{~S} 6 \mathrm{~K} 1$ as well as the expression of SREBP1. However, LRG treatment activated AMPK and suppressed the mTOR-mediated activation of SREBP1, thereby blocking the transcription of target lipogenic genes involved in the liver steatosis of HFD-fed C57 mice. In addition, LRG treatment inhibited HFD-induced nuclear SREBP1 activation, thereby inhibiting SREBP1 translocation into the nucleus and the subsequent changes in liver triglyceride accumulation. 


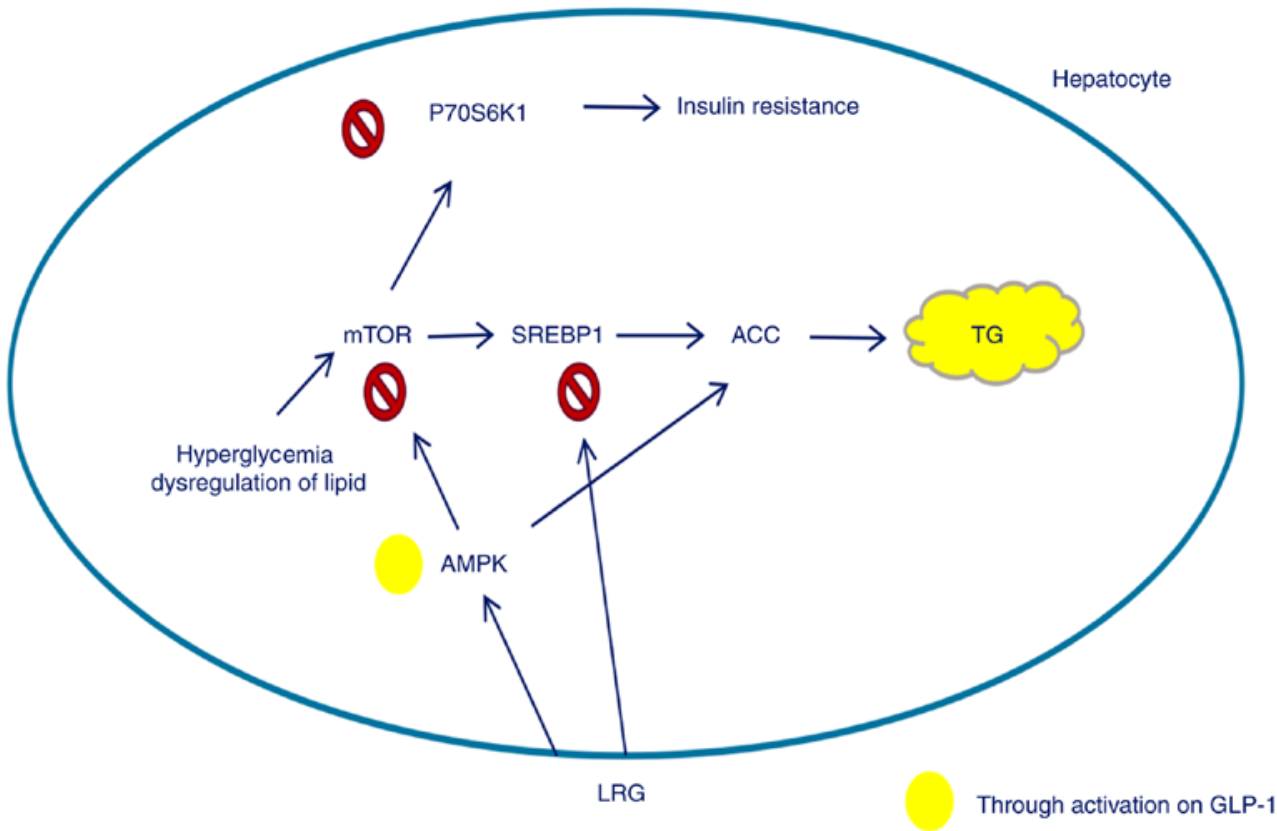

Through inhibition on GLP-1

Role of LRG in the regulation of

AMPK-mTOR-SREBP1 pathway in hepatocytes

Figure 4. Role of LRG in the regulation of the AMPK/mTOR/SREBP1 signaling pathway in NAFLD. LRG inhibits the mTOR/SREBP1/ACC signaling pathway by activating AMPK, thereby regulating the activity of key enzymes involved in lipid synthesis. The protective effect of LRG in NAFLD may be exerted via the activation of the AMPK/mTOR/SREBP1 signaling pathway. LRG, liraglutide; AMPK, adenosine monophosphate-activated protein kinase; mTOR, mechanistic target of rapamycin; SREBP1, sterol regulatory element-binding protein 1; NAFLD, nonalcoholic fatty liver disease; ACC, acetyl coenzyme A carboxylase.
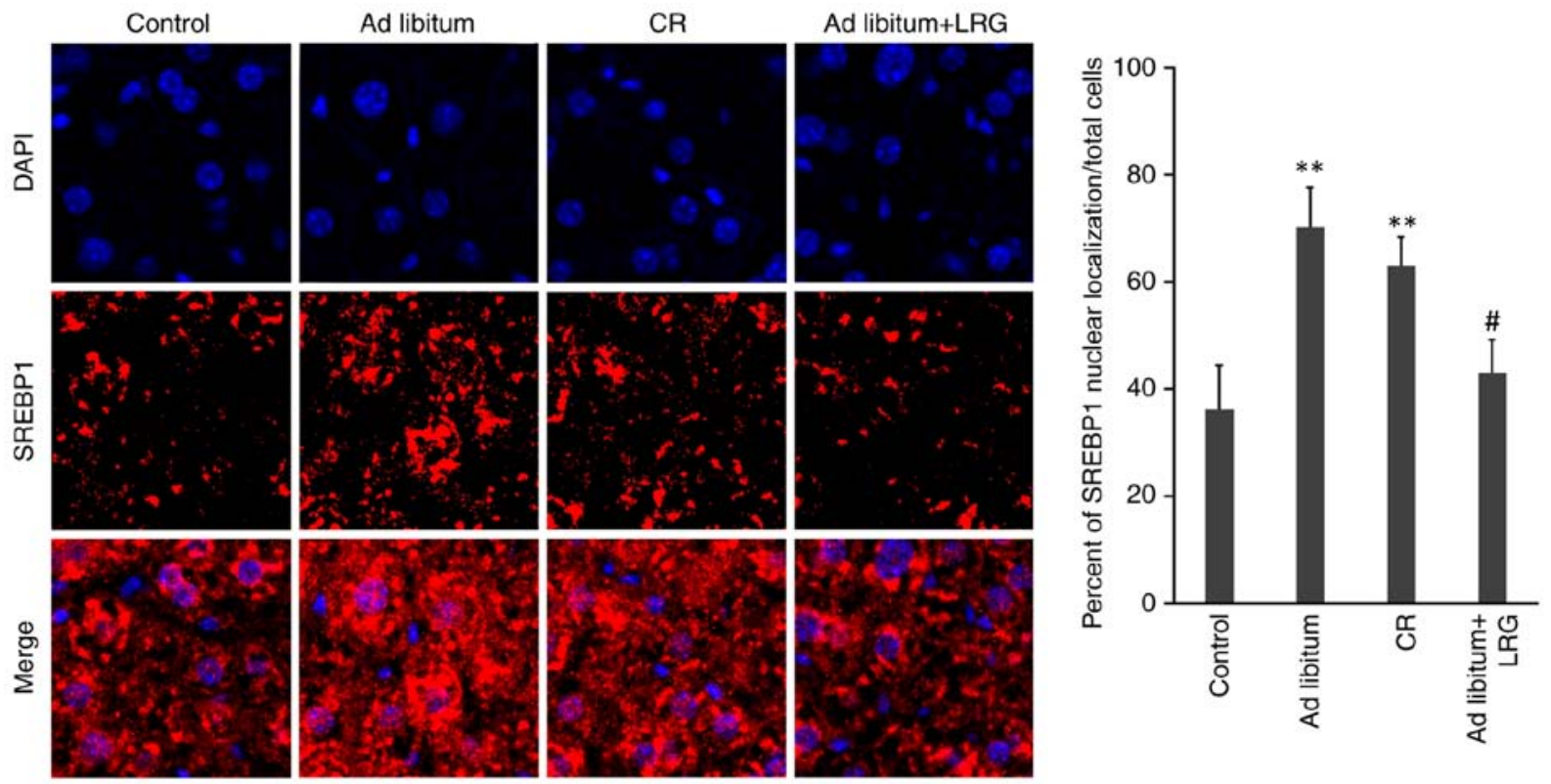

Figure 5. Effects of LRG treatment on the nuclear translocation of SREBP1c in the livers of HFD-fed mice. Immunofluorescence staining was performed to determine the expression and nuclear localization of SREBP1c in hepatocytes from C57/BL6J mice. Magnification, x200. Values are expressed as the mean \pm standard error ( $\mathrm{n}=8 /$ group). ${ }^{* *} \mathrm{P}<0.01$ vs. Control group; ${ }^{~} \mathrm{P}<0.05$ vs. the ad libitum group. LRG, liraglutide; SREBP1c, sterol regulatory element-binding protein 1c; HFD, high-fat diet.

The GLP-1 receptor is widely expressed in various organs of the body and is responsible for improving islet function, suppressing appetite and reducing body weight (39-41). We hypothesized that drug-induced GLP-1 activation may reduce the body weight of HFD-fed mice. Indeed, the body weight changes between the CR group and the group fed HFD 
ad libitum following week 9 were significantly different; however, the biochemical index, molecular expression level and histological features were not significantly different between the two groups. By contrast, the above indicators were significantly different between the CR and LRG groups, indicating that the activation of GLP-1 by LRG directly exerts a pharmacological action on NAFLD in HFD-fed mice.

In conclusion, the GLP-1 receptor agonist LRG reduced the accumulation of hepatic lipids by regulating the AMPK/mTOR/SREBP1 signaling pathway. The present results identified a mechanism by which LRG alleviates hepatic lipid accumulation. Thus, the results of the present study may be used to develop novel therapeutic strategies for steatohepatitis.

\section{Acknowledgements}

The authors would like to thank the Experimental Center of West China Hospital of Sichuan University, West China Animal Research Center and the West China Hospital Department of Endocrinology Experimental Center.

\section{Funding}

No funding was received.

\section{Availability of data and materials}

All data generated or analyzed during this study are included in this published article.

\section{Authors' contributions}

HYC carried out the molecular studies, participated in the immunoassays and drafted the manuscript. SSW carried out the immunoassays. HMT and TH conceived and participated in the design of the study, performed the statistical analysis and helped to draft the manuscript. All authors read and approved the final manuscript.

\section{Ethics approval and consent to participate}

All animal experiments were approved by the Ethics Committee of Sichuan University (Sichuan, China) and were performed in accordance with guidelines of the Institutional Animal Ethics Committee and international guidelines.

\section{Patient consent for publication}

Not applicable.

\section{Competing interests}

The authors declare that they have no conflict of interest.

\section{References}

1. Vernon G, Baranova A and Younossi ZM: Systematic review: The epidemiology and natural history of non-alcoholic fatty liver disease and non-alcoholic steatohepatitis in adults. Aliment Pharmacol Ther 34: 274-285, 2011.
2. Thorn SR, Baquero KC, Newsom SA, El Kasmi KC, Bergman BC, Shulman GI, Grove KL and Friedman JE: Early life exposure to maternal insulin resistance has persistent effects on hepatic NAFLD in juvenile nonhuman primates. Diabetes 63: 2702-2713, 2014.

3. Chackelevicius CM, Gambaro SE, Tiribelli C and Rosso N: Th17 involvement in nonalcoholic fatty liver disease progression to non-alcoholic steatohepatitis. World J Gastroenterol 22: 9096-9103, 2016.

4. Clarke JD, Dzierlenga AL, Nelson NR,Li H, Werts S, Goedken MJ and Cherrington NJ: Mechanism of altered metformin distribution in nonalcoholic steatohepatitis. Diabetes 64: 3305-3313, 2015.

5. Little TJ, Doran S, Meyer JH, Smout AJ, O'Donovan DG, Wu KL, Jones KL, Wishart J, Rayner CK, Horowitz M and Feinle-Bisset C: The release of GLP-1 and ghrelin, but not GIP and CCK, by glucose is dependent upon the length of small intestine exposed. Am J Physiol Endocrinol Metab 291: E647-E655, 2006.

6. Kitade $\mathrm{H}$, Chen G, Ni Y and Ota T: nonalcoholic fatty liver disease and insulin resistance: New insights and potential new treatments. Nutrients 9: pii: E387, 2017.

7. Byrne CD and Targher G: NAFLD: A multisystem disease. J Hepatol 62 (Suppl): S47-S64, 2015.

8. Yamazaki S, Satoh H and Watanabe T: Liraglutide enhances insulin sensitivity by activating AMP-activated protein kinase in male Wistar rats. Endocrinology 155: 3288-3301, 2014.

9. Bakan I and Laplante M: Connecting mTORC1 signaling to SREBP-1 activation. Curr Opin Lipidol 23: 226-234, 2012.

10. Li S, Ogawa W, Emi A, Hayashi K, Senga Y, Nomura K, Hara K, Yu D and Kasuga M: Role of S6K1 in regulation of SREBP1c expression in the liver. Biochem Biophys Res Commun 412: 197-202, 2011.

11. Kim LC, Cook RS and Chen J: mTORC1 and mTORC2 in cancer and the tumor microenvironment. Oncogene 36: 2191-2201, 2017.

12. Song YM, Lee YH, Kim JW, Ham DS, Kang ES, Cha BS, Lee HC and Lee BW: Metformin alleviates hepatosteatosis by restoring SIRT1-mediated autophagy induction via an AMP-activated protein kinase-independent pathway. Autophagy 11: 46-59, 2015

13. He Q, Sha S, Sun L, Zhang J and Dong M: GLP-1 analogue improves hepatic lipid accumulation by inducing autophagy via AMPK/mTOR pathway. Biochem Biophys Res Commun 476: 196-203, 2016.

14. Soliman GA: The integral role of mTOR in lipid metabolism. Cell Cycle 10: 861-862, 2011.

15. Quan HY, Kim DY, Kim SJ, Jo HK, Kim GW and Chung SH: Betulinic acid alleviates non-alcoholic fatty liver by inhibiting SREBP1 activity via the AMPK-mTOR-SREBP signaling pathway. Biochem Pharmacol 85: 1330-1340, 2013.

16. Wang S, Li X, Guo H, Yuan Z, Wang T, Zhang L and Jiang Z: Emodin alleviates hepatic steatosis by inhibiting SREBP1 activity via the CaMKK-AMPK-mTOR-p70S6K signaling pathway: Emodin alleviates NAFLD by CaMKK-AMPK-mTOR-SREBP1. Hepatol Res 47, 2016.

17. Jones B, Bloom SR, Buenaventura T, Tomas A and Rutter GA: Control of insulin secretion by GLP-1. Peptides 100: 75-84, 2018.

18. Ayala JE, Bracy DP, James FD, Julien BM, Wasserman DH and Drucker DJ: The glucagon-like peptide-1 receptor regulates endogenous glucose production and muscle glucose uptake independent of its incretin action. Endocrinology 150: 1155-1164, 2009.

19. Lee J, Hong SW, Rhee EJ and Lee WY: GLP-1 receptor agonist and non-alcoholic fatty liver disease. Diabetes Metab J 36: 262-267, 2012.

20. Butler PC: Glucagon-like peptide 1 drugs as second-line therapy for type 2 diabetes. JAMA Intern Med 176: 1-3, 2016.

21. Wang XC, Gusdon AM, Liu H and Qu S: Effects of glucagon-like peptide-1 receptor agonists on non-alcoholic fatty liver disease and inflammation. World J Gastroenterol 20: 14821-14830, 2014.

22. Dong Y, Lv Q, Li S, Wu Y, Li L, Li J, Zhang F, Sun X and Tong N: Efficacy and safety of glucagon-like peptide-1 receptor agonists in non-alcoholic fatty liver disease: A systematic review and meta-analysis. Clin Res Hepatol Gastroenterol 41: 284-295, 2017.

23. Hansen BC, Gografe S, Pritt S, Jen KC, McWhirter CA, Barman SM, Comuzzie A, Greene M, McNulty JA, Michele DE, et al: Ensuring due process in the IACUC and animal welfare setting: Considerations in developing noncompliance policies and procedures for institutional animal care and use committees and institutional officials. FASEB J 31: 4216-4225, 2017. 
24. Cheluvappa R, Scowen P and Eri R: Ethics of animal research in human disease remediation, its institutional teaching; and alternatives to animal experimentation. Pharmacol Res Perspect 5, 2017.

25. Liu J, Wang G, Jia Y and Xu Y: GLP-1 receptor agonists: Effects on the progression of non-alcoholic fatty liver disease. Diabetes Metab Res Rev 31: 329-335, 2015.

26. Peterson TR, Sengupta SS, Harris TE, Carmack AE, Kang SA Balderas E, Guertin DA, Madden KL, Carpenter AE, Finck BN and Sabatini DM: mTOR complex 1 regulates lipin 1 localization to control the SREBP pathway. Cell 146: 408-420, 2011.

27. Lempiäinen H, Uotila A, Urban J, Dohnal I, Ammerer G, Loewith R and Shore D: Sfp1 interaction with TORC1 and Mrs6 reveals feedback regulation on TOR signaling. Mol Cell 33: 704-716, 2009

28. Gowans GJ, Hawley SA, Ross FA and Hardie DG: AMP is a true physiological regulator of AMP-activated protein kinase by both allosteric activation and enhancing net phosphorylation. Cell Metab 18: 556-566, 2013.

29. Bojic LA, Telford DE, Fullerton MD, Ford RJ, Sutherland BG, Edwards JY, Sawyez CG, Gros R, Kemp BE, Steinberg GR and Huff MW: PPARס activation attenuates hepatic steatosis in Ldlr-/- mice by enhanced fat oxidation, reduced lipogenesis, and improved insulin sensitivity. J Lipid Res 55: 1254-1266, 2014.

30. Laplante M and Sabatini DM: An emerging role of mTOR in lipid biosynthesis. Curr Biol 19: R1046-R1052, 2009.

31. Melnik BC: Linking diet to acne metabolomics, inflammation, and comedogenesis: An update. Clin Cosmet Investig Dermatol 8: 371-388, 2015.

32. Carling D and Viollet B: Beyond energy homeostasis: The expanding role of AMP-activated protein kinase in regulating metabolism. Cell Metab 21: 799-804, 2015.
33. Jeong KJ, Kim GW and Chung SH: AMP-activated protein kinase: An emerging target for ginseng. J Ginseng Res 38: 83-88, 2014.

34. Horton JD, Goldstein JL and Brown MS: SREBPs: Activators of the complete program of cholesterol and fatty acid synthesis in the liver. J Clin Invest 109: 1125-1131, 2002.

35. Düvel K, Yecies JL, Menon S, Raman P, Lipovsky AI, Souza AL, Triantafellow E, Ma Q, Gorski R, Cleaver S, et al: Activation of a metabolic gene regulatory network downstream of mTOR complex 1. Mol Cell 39: 171-183, 2010.

36. Kelleher AR, Kimball SR, Dennis MD, Schilder RJ and Jefferson LS: The mTORC1 signaling repressors REDD1/2 are rapidly induced and activation of p70S6K1 by leucine is defective in skeletal muscle of an immobilized rat hindlimb. Am J Physiol Endocrinol Metab 304: E229-E236, 2013.

37. Ricoult SJ, Yecies JL, Ben-Sahra I and Manning BD: Oncogenic $\mathrm{PI} 3 \mathrm{~K}$ and K-Ras stimulate de novo lipid synthesis through mTORC1 and SREBP. Oncogene 35: 1250-1260, 2016.

38. Laplante M and Sabatini DM: Regulation of mTORC1 and its impact on gene expression at a glance. J Cell Sci 126: 1713-1719, 2013.

39. Katsurada $\mathrm{K}$ and Yada T: Neural effects of gut- and brain-derived glucagon-like peptide-1 and its receptor agonist. J Diabetes Investig 7 (Suppl 1): S64-S69, 2016.

40. Cariou B: Pleiotropic effects of insulin and GLP-1 receptor agonists: Potential benefits of the association. Diabetes Metab 41 (6 Suppl 1): 6S28-6S35, 2015.

41. Seufert J and Gallwitz B: The extra-pancreatic effects of GLP-1 receptor agonists: A focus on the cardiovascular, gastrointestinal and central nervous systems. Diabetes Obes Metab 16: 673-688, 2014. 